\title{
Lymphoepithelioma-Like Carcinoma of Urinary Bladder with Diagnostic Dilemma: Case Report of a Rare Variant of Urothelial Carcinoma
}

\author{
Swati Saini ${ }^{1}$ Monika Garg ${ }^{1}$ Shaina Goyal ${ }^{1} \quad$ Anita Chaudhary ${ }^{1}$ \\ ${ }^{1}$ Department of Pathology, Government Medical College, Patiala, \\ Punjab, India \\ Address for correspondence Swati Saini, MD, Department of Pathology, \\ Government Medical College, Patiala, 147001, Punjab, India \\ (e-mail: 87dr.swati@gmail.com). \\ J Lab Physicians 2022;14:84-86.
}

\begin{abstract}
Lymphoepithelioma-like carcinoma of the urinary bladder is a rare variant of urothelial carcinoma that was first described by Zukerberg in 1991 and confirmed as a type of urothelial carcinoma by the WHO classification of the urinary system tumors. A 63-year-old man presented with the chief complaint of gross hematuria for 2 months. Sections from transurethral resection of the bladder revealed urothelial carcinoma. A radical cystoprostatectomy was performed and a final histopathological examination revealed high-grade

Keywords

- bladder

- carcinoma

- lymphoepithelioma

- urothelial muscle-invasive urothelial carcinoma, lymphoepithelial variant. The patient has been receiving adjuvant chemotherapy with no recurrence reported to date. This is the third case reported in India to date to the best of our knowledge. Owing to its rare presentation, no definite treatment guidelines have been established for this entity. Various studies from the English literature suggest a conservative approach.
\end{abstract}

\section{Introduction}

Lymphoepithelioma depicts an undifferentiated carcinoma of the nasopharynx characterized microscopically by the predominance of lymphoid aggregates in the background. Carcinomas with similar histological features arising outside the nasopharynx are known as LELC, which have been reported in various organs such as the thymus, cervix, and salivary glands. ${ }^{1}$ Involvement of urinary bladder was first reported by Zukerberg et al in 1991 with a reported incidence between $0.4 \%$ and $1.3 \%$ of all bladder carcinomas. ${ }^{2}$ It has been categorized into three subgroups: pure $(100 \%$ lymphoepithelioma component), predominant (between $50 \%$ and $100 \%)$, and focal $(<50 \%)^{3}$ Owing to the scarcity of the case in the English literature, we hereby present a case of pure LELC bladder and discuss important differential diagnosis, various treatment modalities, and prognosis of this rare tumor.

\section{Case Report}

A 63-year-old male patient presented with a complaint of painless gross hematuria. He had no prior personal/family medical history of this complaint. There were no notable findings in blood tests and chest radiography. Urine cytology results were negative. A CECT abdomen was performed that revealed a tumor arising from the left anterolateral wall of the urinary bladder projecting into its lumen with a small exophytic component reaching beyond the serosal surface on the inferior aspect (-Fig. 1). TURBT revealed urothelial carcinoma. Radical cystoprostatectomy was performed. Pathological findings revealed extensively published online November 23, 2021
DOI https://doi.org/ 10.1055/s-0041-1736481. ISSN 0974-2727. (c) 2021. The Indian Association of Laboratory Physicians. All rights reserved.

This is an open access article published by Thieme under the terms of the Creative Commons Attribution-NonDerivative-NonCommercial-License, permitting copying and reproduction so long as the original work is given appropriate credit. Contents may not be used for commercial purposes, or adapted, remixed, transformed or built upon. (https://creativecommons.org/ licenses/by-nc-nd/4.0/)

Thieme Medical and Scientific Publishers Pvt. Ltd., A-12, 2nd Floor, Sector 2, Noida-201301 UP, India 


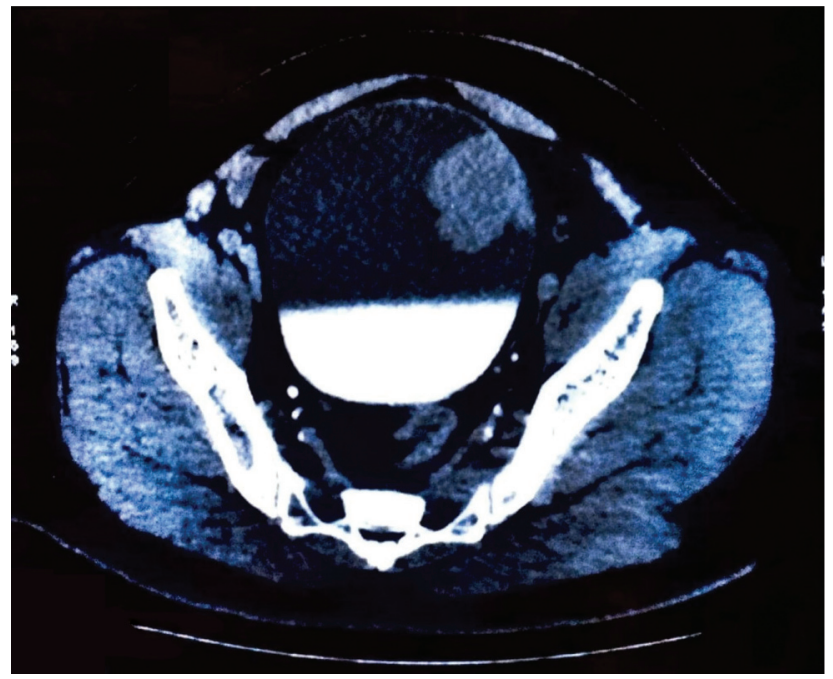

Fig. 1 A CECT abdomen revealing a tumor arising from the left anterolateral wall of the urinary bladder, projecting into its lumen with a small exophytic component reaching beyond the serosal surface on the inferior aspect.

infiltrative tumor cells with pleomorphic nuclei and prominent nucleoli, interspersed with numerous lymphocytes, eosinophils, and mast cells and invading the muscle and reaching close to the serosa and perivesical fat (-Figs. 2 and 3). Perineural invasion was also noted. The final pathological diagnosis was urothelial carcinoma, high grade, lymphoepithelial variant, pT3a. Tumor cells were positive for CK7 and negative for CK20, p63, and GATA3 (-Fig. 4). An adjuvant chemotherapy was initiated and cancer recurrence was not apparent at usual follow-up as determined by CT scan.

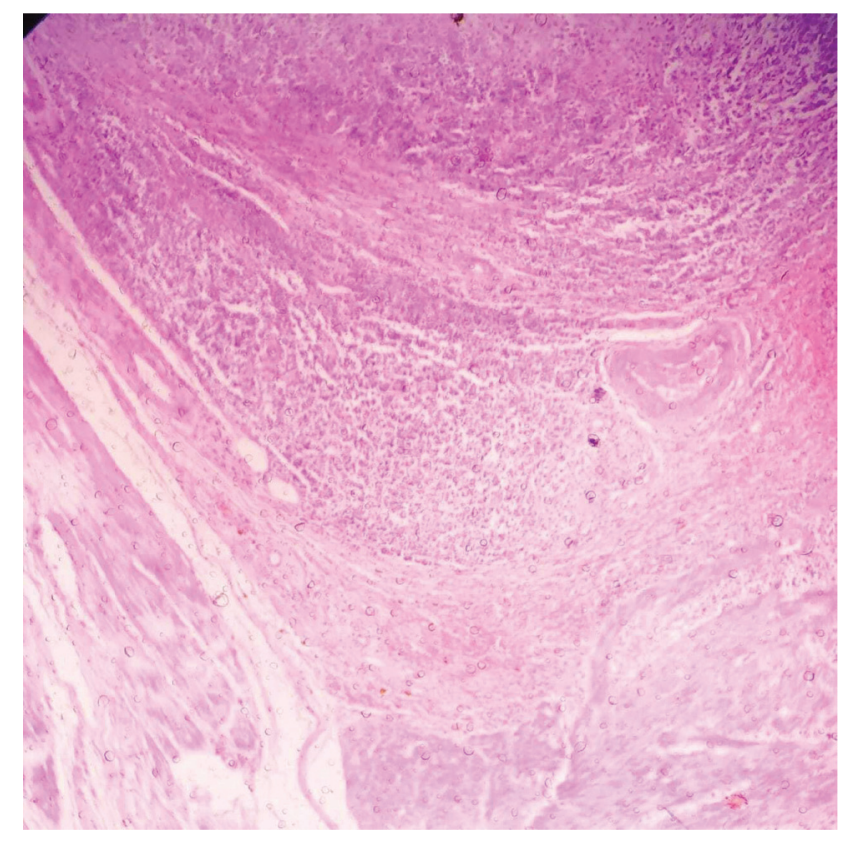

Fig. 2 Microphotograph showing epithelial tumor cells mixed with lymphoid cells $(H \& E, \times 100)$.

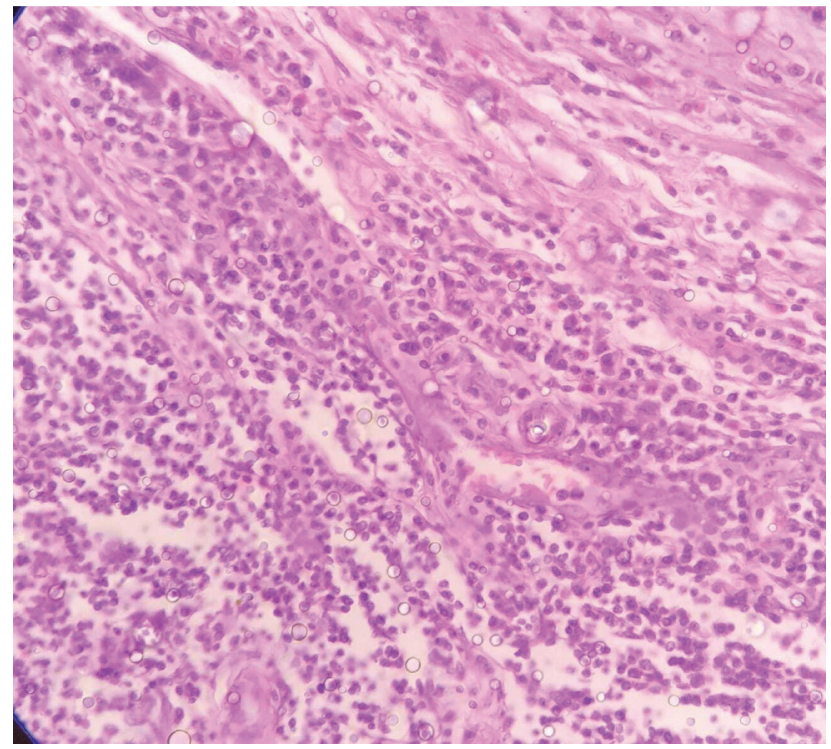

Fig. 3 Undifferentiated tumor cells with syncytial appearance invading the muscles. Lymphoid cells are also seen (H\&E, $\times 400)$.

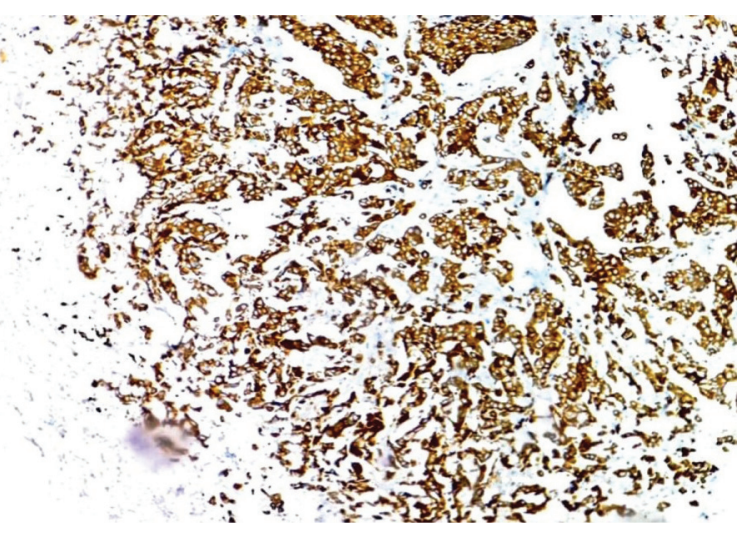

Fig. 4 Immunohistochemistry showing intense cytoplasmic and membrane positivity of CK7 in tumor cells (Immunohistochemistry, CK7, ×400).

\section{Discussion}

According to 2016 WHO classification, LELC is a rare variant of urothelial carcinoma with a reported incidence between $0.4 \%$ and $1.3 \%$ of all bladder carcinomas. ${ }^{4}$ The majority of patients present in late adulthood and are at stage T2-T3 at diagnosis. ${ }^{4}$ Histological features of LELC closely resemble nasopharyngeal lymphoepithelioma, with the tumor growing in the nests, sheets, or cords of large undifferentiated malignant epithelial cells in the background of dense inflammatory infiltrate comprising mainly lymphocytes, plasma cells, and a few eosinophils and neutrophils. ${ }^{1}$ Amin et al categorized LELC of the urinary bladder into three subgroups based on lymphoepithelioma component: pure (100\%), predominant $(>50 \%)$, and focal $(<50 \%)$. These subgroups significantly determine the prognosis as disease-free survival is higher in pure and predominant LELCB than in mixed type. ${ }^{3}$ Pure LELCB must be differentiated from lymphoma or reactive inflammatory lesion, small cell carcinoma, poorly 
differentiated urothelial carcinoma with lymphoid cell infiltration. ${ }^{5}$ Immunohistochemistry for cytokeratin and lymphoid markers can help in eliminating the possibility of lymphoma. ${ }^{1}$ Reactive inflammatory lesion can be excluded using immunohistochemistry. In the present case, the tumor cells revealed positivity for CK7 and negativity for CK20, p63, and GATA3 markers confirming the urothelial origin of the tumor. Due to the paucity of the cases described, there are currently no standard treatment protocols for LELCB. Some reports described pure and predominant LELCB as being more sensitive to chemotherapy than conventional urothelial carcinoma; ${ }^{4}$ this provides a potential to salvage bladder function. Focal LELCB is best managed by multifocal therapies including radical cystectomy, EBRT, and adjuvant systemic chemotherapy owing to its more aggressive nature. ${ }^{6}$ Our case underwent radical cystoprostatectomy with chemotherapy and was free of disease 8 months after the surgery.

\section{Conclusion}

LELCB is a rare tumor that occurs in older males. No standard management protocols have been established. On account of this, reporting of this variant would add to the caveat in literature and help clinicians to devise appropriate treatment protocols.

Conflict of Interest

None declared.

\section{References}

1 Singh NG, Mannan AA, Rifaat AA, Kahvic M. Lymphoepitheliomalike carcinoma of the urinary bladder: report of a rare case. Ann Saudi Med 2009;29(06):478-481

2 Zukerberg LR, Harris NL, Young RH. Carcinomas of the urinary bladder simulating malignant lymphoma. A report of five cases. Am J Surg Pathol 1991;15(06):569-576

3 Amin MB, Ro JY, Lee KM, et al. Lymphoepithelioma-like carcinoma of the urinary bladder. Am J Surg Pathol 1994;18(05):466-473

4 Tamas EF, Nielsen ME, Schoenberg MP, Epstein JI. Lymphoepithelioma-like carcinoma of the urinary tract: a clinicopathological study of 30 pure and mixed cases. Mod Pathol 2007;20(08): 828-834

5 Raphael V, Jitani AK, Sailo SL, Vakha M. Lymphoepithelioma-like carcinoma of the urinary bladder: a rare case report. Urol Ann 2015;7(04):516-519

6 Kushida N, Kushakabe T, Kataoka M, Kumagai S, Aikawa K, Kojima Y. External beam radiotherapy for focal lymphoepithelioma-like carcinoma in the urinary bladder: a case report and literature review. Case Rep Oncol 2015;8(01):15-20 